\title{
The surjectivity problem for one-generator, one-relator extensions of torsion-free groups
}

\author{
Marshall M COHEN \\ COLIN ROURKe \\ Cornell University, Ithaca NY, 14853-4102, USA \\ and \\ Warwick University, Coventry, CV4 7AL, UK \\ Email: marshall@math.cornell.edu and cpr@maths.warwick.ac.uk
}

\begin{abstract}
We prove that the natural map $G \rightarrow \widehat{G}$, where $G$ is a torsion-free group and $\widehat{G}$ is obtained by adding a new generator $t$ and a new relator $w$, is surjective only if $w$ is conjugate to $g t$ or $g t^{-1}$ where $g \in G$. This solves a special case of the surjectivity problem for group extensions, raised by Cohen [2].
\end{abstract}

AMS Classification numbers Primary: 20E22, 20F05

Secondary: 57M20, 57Q10

Keywords: Surjectivity problem, torsion-free groups, Whitehead torsion, Kervaire conjecture

Proposed: Cameron Gordon

Seconded: Joan Birman, Wolfgang Metzler

(C) Geometry $\& \mathcal{T}$ Topology $\mathcal{P}$ ublications
Received: 12 September 2000 Accepted: 6 March 2001 


\section{Introduction}

In this paper we prove the following theorem.

Main Theorem Suppose that $G$ is a torsion-free group and that $\langle t\rangle$ is an infinite cyclic group with generator $t$. Let $w$ be an element of the free product $G *\langle t\rangle$ and let $\langle\langle w\rangle\rangle$ be the normal subgroup of $G *\langle t\rangle$ generated by w. View $G$ as a subgroup of $G *\langle t\rangle$ and let $i$ be the inclusion $G \longrightarrow G *\langle t\rangle$. Consider the natural homomorphism

$$
q=\pi i: \quad G \stackrel{i}{\longrightarrow} G *\langle t\rangle \stackrel{\pi}{\longrightarrow} \widehat{G}=\frac{G *\langle t\rangle}{\langle\langle w\rangle\rangle} .
$$

If $q$ is onto then $w$ is conjugate to $g t$ or $g t^{-1}$ for some $g \in G$.

There are standard ways in which this algebraic situation may be realized topologically. These lead to the following results.

Corollary 1 Suppose that $L$ is a connected $C W$ complex with torsion-free fundamental group and that the $C W$ complex $\widehat{L}=L \cup e^{1} \cup e^{2}$ is constructed by attaching a 1 -cell to $L$ and a $2-$ cell to $L \cup e^{1}$. If the inclusion map $j: L \longrightarrow \widehat{L}$ induces a surjection $j_{*}: \pi_{1} L \longrightarrow \pi_{1} \widehat{L}$ then $j$ is a simple-homotopy equivalence.

Proof This follows from elementary facts about the invariance of Whitehead torsion under homotopy of attaching maps [1; Section 5] and the fact that if $\widehat{L}=L \cup e^{1} \cup e^{2}$, where $e^{1}$ is a circle and $e^{2}$ is a 2 -cell attached by a word $g t$, then $e^{1}$ is a free face of $\widehat{L}$ and $\widehat{L}$ collapses to $L$ by an elementary collapse.

Corollary 2 Suppose that $M$ is a connected $n$-manifold with torsion free fundamental group and that $\left(W, M, M^{\prime}\right)$ is an $h$-cobordism with exactly one handle of index one and one handle of index two and no other handles (or dually with exactly one $n$-handle and one $(n-1)$-handle). Then $\left(W, M, M^{\prime}\right)$ is an $\mathrm{s}$-cobordism.

Proof This is a consequence of the fact that a $k$-handle $D^{k} \times D^{n+1-k}$ collapses to its core union its attaching tube, $D^{k} \times\{0\} \cup \partial D^{k} \times D^{n+1-k}$, see eg [10; Chapter 6]. So the CW theory applies to the handlebody theory.

\section{Background}

The surjectivity problem for group extensions and the question of which Whitehead torsions can be realized were formulated by Cohen [2] and Metzler [6]; for more details on these problems and the relevance of our results, see Section 5.

It will be useful to note from the outset that the conclusion of the main theorem may be restated according to the following lemma. 
Lemma 1 If $G \subset G *\langle t\rangle \stackrel{\pi}{\longrightarrow} G *\langle t\rangle /\langle\langle W\rangle\rangle$ where $W$ is a set of words in $G *\langle t\rangle$ then $q=\pi \mid G$ is onto $\Longleftrightarrow g t$ lies in the kernel of $\pi$ for some $g \in G$.

Proof $q$ is onto $\Longleftrightarrow[\pi(t) \in \pi(G)] \Longleftrightarrow\left[\pi(t)=\pi\left(g^{-1}\right)\right.$ for some $g \in$ $G] \Longleftrightarrow[\pi(g t)=1] \Longleftrightarrow[g t \in \operatorname{kernel}(\pi)$ for some $g \in G]$.

Any element $w \in G *\langle t\rangle$ has a unique expression as a reduced word, $w=$ $g_{0} t^{q_{1}} g_{1} t^{q_{2}} \ldots g_{n-1} t^{q_{n}} g_{n}$, where $g_{i} \in G$ are non-trivial for $0<i<n$ and $q_{i}$ are non-zero integers for each $i$. The word $w$ is cyclically reduced if further $g_{n}=1$ and if $n>1$ then $g_{0} \neq 1$. Up to cyclic permutation there is a unique cyclically reduced word in the conjugacy class of $w$, see eg [3, Proposition 3.9]. Since $\widehat{G}$ depends only the conjugacy class of $w$, there is no loss in assuming that $w$ is cyclically reduced and we shall do so without comment from now on. We call $\sum_{i=1}^{n} q_{i}$ the exponent sum of $t$ in $w$, denoted $\operatorname{ex}(w)$. The unreduced word $t^{q_{1}} t^{q_{2}} \ldots t^{q_{n}}$ is called the $t$-shape of $w$ and, thinking of $w=1$ as an equation over $G$, we call the elements $g_{i}$ the coefficients of $w$.

It is easy to see that if $q: G \longrightarrow \widehat{G}$ is surjective then $\operatorname{ex}(w)= \pm 1$, since otherwise the abelianization of $\widehat{G} /(q(G)=1)$ will be non-trivial. So, replacing $w$ by $w^{-1}$ if necessary, we may assume in our discussion that $\operatorname{ex}(w)=1$. Under this hypothesis Klyachko [8], in 1993, gave a brilliant argument to prove the following theorem, which implies the Kervaire conjecture [7] in the case where $G$ is torsion-free.

Theorem (Klyachko) If $G$ is a torsion-free group and $w \in G *\langle t\rangle$ with $\operatorname{ex}(w)=1$ then the natural homomorphism $q: G \longrightarrow \widehat{G}=\frac{G *\langle t\rangle}{\langle\langle w\rangle\rangle}$ is injective.

An exposition (and extension) of Klyachko's theorem was given by Fenn and Rourke [4] in 1996. To prove our theorem we will use Klyachko's result and his method, following closely the exposition in [4]. We will quote some definitions and results from [4] and give those proofs in detail for which the arguments differ and for which (proving the contrapositive) the hypothesis is used that $w$ is not conjugate to $g t$ for any $g \in G$.

\section{Outline of the paper}

In Section 2 we consider a group $\Gamma$ in a slightly more general situation than $G$ above. We assume (contrary to our Main Theorem) that $w$ is not conjugate to $g t$ for any $g \in \Gamma$ but that some $g t$ is in the kernel of $\Gamma *\langle t\rangle \longrightarrow \widehat{\Gamma}$. We show how to construct a certain non-trivial CW subdivision of the 2-sphere, with edges labelled $t^{ \pm 1}$ and all but one corner labelled by an element of $\Gamma$. 
In Section 3 we prove our main theorem in a special case: We denote $g^{t}=t^{-1} g t$. If $w$ has the form $w=b_{0} a_{0}^{t} b_{1} a_{1}^{t} \ldots b_{r} a_{r}^{t} c t$, where the $a_{i}, b_{i}$ and $c$ are all elements of $G$ and $b_{0} a_{0}^{t} b_{1} a_{1}^{t} \ldots b_{r} a_{r}^{t} c \notin G$ then $q: G \longrightarrow \widehat{G}$ is not onto.

In Section 4, we complete the proof of the main theorem. We use an algebraic trick to parlay the result of Section 4 into a proof that, in general, if $\operatorname{ex}(w)=1$ and $w$ is not conjugate to $g t$ for any $g \in G$ then $q: G \longrightarrow \widehat{G}$ is not onto.

In Section 5 we briefly discuss the general surjectivity problem, in which $n$ generators and $n$ relators are added to a group $G$. We give a bit of history and comment on the relevance of our result for $n=1$ to the general problem.

Finally, in Section 6 we extend our result to prove that if $w \in G *\langle t\rangle$ is a word not of the form $g t$ whose $t$-shape is amenable (see $[4,5])$ then no word with $t$-shape $t^{n}$ can be in the kernel of $\pi: G \rightarrow \widehat{G}=\frac{G *\langle t\rangle}{\langle\langle w\rangle\rangle}$.

\section{The cell subdivision lemma}

In this section we prove the cell subdivision lemma (below) which is modelled on [4; Lemma 3.2].

The lemma uses the idea of a corner of a 2-cell in a cell subdivision $K$ of the $2-$ sphere. This can be regarded as the (oriented) angle formed by the two adjacent edges meeting at a vertex (0-cell) in the boundary of the $2-$ cell. If all the corners of a 2-cell are labelled by elements of a group, then a word can be read around the 2-cell boundary by composing these elements either unchanged or inverted according as the orientation of the corner agrees or dissagrees with that of the 2 -cell boundary. Similarly if all the corners at a vertex are labelled then a word can be read around that vertex. We shall always orient corners clockwise, thus if the above words are read clockwise for vertices and anticlockwise for 2-cells, then no inversion is necessary. See figure 1 for an example: the word read around the boundary is $a b c$; after insertion of $t$ or $t^{-1}$ at the arrows (see part (e) of the lemma below) it reads $t a t^{-1} b t^{-1} c$.

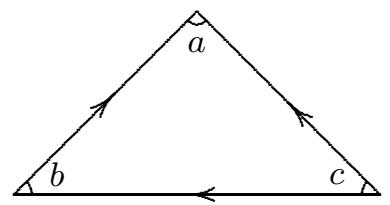

Figure 1: Reading the boundary of a 2-cell: $t a t^{-1} b t^{-1} c$ 
Let $H$ be a subgroup of a group $\Gamma$ and let $g \in \Gamma$. We say that $g$ is free relative to $H$ if the subgroup $\langle g, H\rangle$ of $\Gamma$ generated by $g$ and $H$ is naturally the free product $\langle g\rangle * H$ of an infinite cyclic group $\langle g\rangle$ with $H$. (Note in particular that $g$ has infinite order.)

If $g, h$ are elements of a group let $g^{h}$ denote $h^{-1} g h$.

In this section and the next, we shall consider the following working hypotheses:

\section{Working hypotheses}

Suppose that $H$ and $H^{\prime}$ are two isomorphic subgroups of a group $\Gamma$ under the isomorphism $h \rightarrow h^{\phi}, h \in H$. Suppose that for each $i, a_{i}, b_{i}$ are elements of $\Gamma$ such that $a_{i}$ is free relative to $H$ and $b_{i}$ is free relative to $H^{\prime}$. Let $c$ be an arbitary element of $\Gamma$.

Let $w_{0}$ be the word

$$
b_{0} a_{0}^{t} b_{1} a_{1}^{t} b_{2} a_{2}^{t} \cdots b_{r} a_{r}^{t} c t
$$

in $\Gamma *\langle t\rangle$, where $r \geq 0$, and and let $W \subset \Gamma *\langle t\rangle$ be the set of words $\left\{w_{0}, h^{t}\left(h^{\phi}\right)^{-1} \mid h \in H\right\}$. Let $\langle\langle W\rangle\rangle$ be the normal closure of $W$ in $\Gamma *\langle t\rangle$ and let $\widehat{\Gamma}$ denote $(\Gamma *\langle t\rangle) /\langle\langle W\rangle\rangle$.

\section{Cell subdivision lemma}

Assume the working hypotheses, above. Suppose that, for some $g \in \Gamma$, gt is in the kernel of the natural map $\Gamma *\langle t\rangle \rightarrow \widehat{\Gamma}$.

Then there is a cell subdivision $K$ of the 2 -sphere such that

(a) the edges (1-cells) of $K$ are oriented,

(b) the corners (all oriented clockwise) are labelled by coefficients of elements $w$ or $w^{-1}$ for $w \in W$, with the exception of one particular corner at one particular vertex $v_{0}$ which is unlabelled,

(c) the clockwise product of the corner labelling around any vertex is $1 \in \Gamma$ except for $v_{0}$ where it is undefined,

(d) there is one special 2-cell $e_{\infty}^{2}$ which contains the unlabelled corner and has boundary a single edge and the single vertex $v_{0}$,

(e) with the exception of $e_{\infty}^{2}$, the corner labels of any 2-cell (in anticlockwise order) are the coefficients of $w$ or $w^{-1}$ for some $w \in W$ (up to cyclic rotation) with the property that, if on passing from one corner to an adjacent corner the element $t$ or $t^{-1}$ is inserted according to whether the 
intervening edge is oriented in the same or opposite direction (see figure $1)$, then the whole of $w$ or $w^{-1}$ is recovered,

(f) the cell decomposition is irreducible in the following senses:

type (1) there do not exist two 2-cells with an edge in common (necessarily read as $t$ in one and $t^{-1}$ in the other) such that, starting with one vertex of this edge, the words read in these 2-cells are inverses of each other,

type (2) there does not exist a chain of 2-gons with common vertices $a, b$ such that the product of the corner labels in the chain at $a$ (or, equivalently, at $b$ ) is $1 \in \Gamma$,

(g) the cell subdivision is non-degenerate in that there exist at least two vertices and at least three 2-cells; in particular there is a cell $e_{1}^{2} \neq e_{\infty}^{2}$ whose boundary contains $\partial e_{\infty}^{2}$ as a proper subset (see figure 5).

Proof The proof uses transversality as in the proofs of [4; Lemmas 3.1 and $3.2]$.

Choose a 2-complex $L$ with $\pi_{1}(L)=\Gamma$ and form the $2-$ complex $\widehat{L}$ with $\pi_{1}(\widehat{L})=$ $\widehat{\Gamma}$ by attaching a 1 -cell $\gamma$ to the base point $*$ of $L$ (corresponding to $t$ ) and a 2-cell $\sigma_{w}$ with attaching map determined by $w$ for each $w \in W$.

Since $g t$ is trivial in $\pi_{1}(\widehat{L})$ there is a map of a 2-disc $f: D^{2} \rightarrow \widehat{L}$ whose boundary maps to $L \cup \gamma$ and which represents $g t \in \pi_{1}(L \cup \gamma)=\Gamma *\langle t\rangle$. Make $f$ transverse to the centres of the $2-$ cells $\sigma_{w}$. It follows that the inverse images of small neighbourhoods of these centres is a collection of disjoint discs $D_{1}, \ldots, D_{m}$ in the interior of $D^{2}$. By a radial expansion of $f$ on these discs we may assume that their image is the whole of one of the $\sigma_{w}$. It follows that the punctured $\operatorname{disc} P=\operatorname{closure}\left(D^{2}-\left(D_{1} \cup \cdots \cup D_{m}\right)\right)$ is mapped by $f$ to $L \cup \gamma$. Let $p$ be the centre of $\gamma$. Make $f \mid P$ transverse to $p$. Then $f^{-1} p$ is a 1 -manifold $Z$ properly embedded in $P$. By a radial expansion along $\gamma$ we can assume that $Z$ has a neighbourhood $N$ which is a normal $I$-bundle, where each fibre is mapped by $f$ to $\gamma$ and closure $(P-N)$ is mapped by $f$ to $L$.

We now simplify the subset $\mathcal{H}_{f}=D_{1} \cup \cdots \cup D_{m} \cup N$ of $D^{2}$ as follows. Suppose $N$ contains an annulus component $\mathcal{A}$ in the interior of $P$. Let $D^{\prime}$ denote the interior disc of $D^{2}$ which bounds the interior boundary component of the annulus. Then $D^{\prime \prime}=D^{\prime} \cup \mathcal{A}$ is a sub disc of $D^{2}$ whose boundary is mapped to $*$ by $f$. We can then redefine $f$ so that $f\left(D^{\prime \prime}\right)=*$ leaving $f$ unchanged outside $D^{\prime \prime}$.

At this point $\mathcal{H}_{f}$ can be regarded as a collection of 0 -handles (the $D_{i}$ ) and 1-handles (the components of $N$ ) attached to the 0 -handles and to $\partial D^{2}$ (in fact there is precisely one 1-handle attached to $\partial D^{2}$ by one end) see figure 2 . 


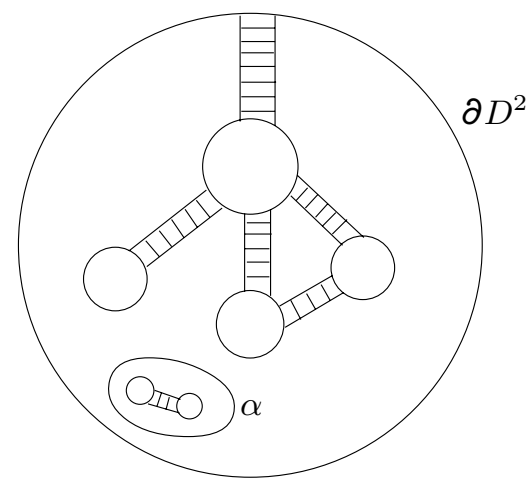

Figure 2: A view of $\mathcal{H}_{f}$

We now prove that we may assume that $\mathcal{H}_{f}$ is connected. Suppose not. Choose an innermost component $C$. Draw a simple loop $\alpha$ around $C$ separating it from the rest of $\mathcal{H}_{f}$. Up to conjugacy $\alpha$ represents an element of $\pi_{1}(L)=\Gamma$ which is trivial in $\pi_{1}(\widehat{L})=\widehat{\Gamma}$. But Klyachko proves that $\Gamma$ injects in $\widehat{\Gamma}$ (this is the precise content of [4; Theorem 4.1, page 62]) and hence we may redefine $f$ so that the inside of $\alpha$ is mapped to $L$, which simplifies $\mathcal{H}_{f}$.

Note that the 0 -handles can be labelled by elements $w$ or $w^{-1}$ for $w \in W$ according to the corresponding 2 -cell of $\widehat{L}$ and orientation. We say that $\mathcal{H}_{f}$ is type (1) reducible if there is a pair of 0 -handles labelled by $w$ and $w^{-1}$ (the same $w$ ) and joined by a 1-handle which represents the same occurrence of $t$ (respectively $t^{-1}$ ) in each word. In this situation we can again simplify $\mathcal{H}_{f}$ without changing $f \mid \partial D^{2}$ by redefining $f$ near these 0 -handles and joining 1 -handle (see figure 3 ).
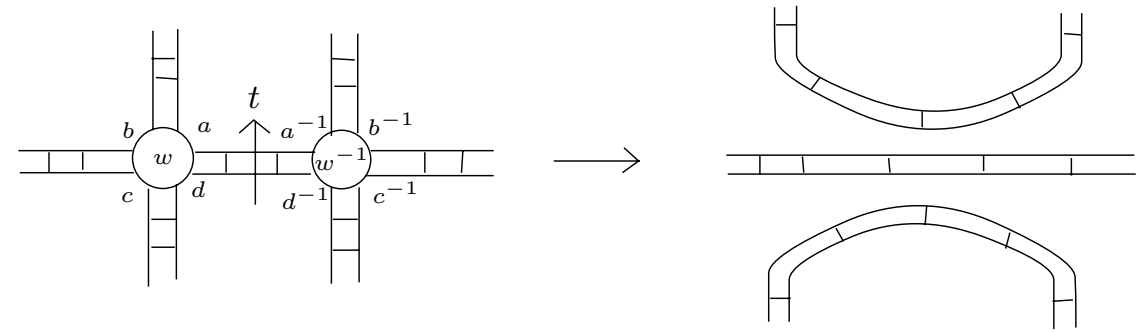

Figure 3: Type (1) reduction of $\mathcal{H}_{f}$

We say that $\mathcal{H}_{f}$ is type (2) reducible if there is a chain of 0 -handles (each having two 1-handles attached to it) labelled by words $h_{i}^{t}\left(h_{i}^{\phi}\right)^{-1}, i=1,2 \ldots, q$ with $h_{1} h_{2} \ldots h_{q}=1$ in $\Gamma$ (figure 4 ). 


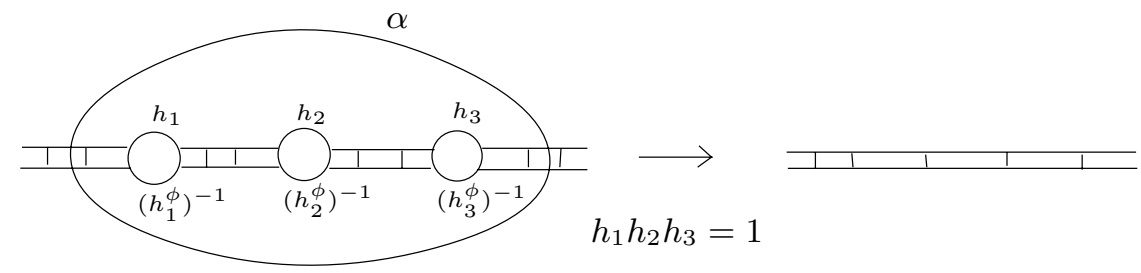

Figure 4: Type (2) reduction of $\mathcal{H}_{f}$

If the chain forms a loop, the handlebody is not connected and this chain and everything inside it may be eliminated as indicated earlier. Otherwise, the curve $\alpha$ indicated in figure 4 maps to $t t^{-1}$ in $\Gamma *\langle t\rangle$ and there is another simplification given by omitting this chain of 0 -handles and redefining $f$ inside $\alpha$ using the null-homotopy of $t t^{-1}$ in $L \cup \gamma$. After these simplifications there may now be more simplifications of the first two types which can be performed. Repeat all four until no more are possible, Thus we can assume that $\mathcal{H}_{f}$ is connected and irreducible.

We now extend $\mathcal{H}_{f}$ to a handle decomposition $\mathcal{H}$ of $S^{2} \supset D^{2}$ by letting the outside of $D^{2}$ be one 0 -handle (denoted $h_{\infty}^{2}$ ) and the regions of $D^{2}-\mathcal{H}_{f}$ be the 2 -handles.

The required 2 -complex $K$ is the dual complex to $\mathcal{H}$ obtained by putting a vertex inside each 2 -handle and joining by an edge across each 1 -handle. The outside $2-$ cell is $e_{\infty}^{2}$ (containing $h_{\infty}^{2}$ ) and has boundary containing a single vertex $v_{0}$. Corners of 2-cells other than this corner are labelled by the coefficient of the word $w$ or $w^{-1}$ labelling the 0 -handle inside the 2-cell opposite the corner. See figure 5 .

The required properties of $K$ all follow from the construction: 1-cells are oriented by the orientations of the $I$-bundles (1-handles) that they cross and properties (a) to (e) follow at once (the word read around the boundary of a 2-cell is the label on the contained 0-handle). Property (f) follows from the irreducibility of $\mathcal{H}_{f}$.

Finally, property (g) uses the hypothesis that $r \geq 0$ (ie, that $w_{0}$ is not conjugate to $g t$ for any $g \in \Gamma$ ). In order that every 1 -handle of $\mathcal{H}$ have each end on some $\partial D_{i}^{2}$, except that one of them has one end on $\partial D^{2}$, at least one of the 0 -handles $D_{i}^{2}$ must be a $w_{0}$ or $w_{0}^{-1}$ handle. Because $r \geq 0$, this handle must have at least three 1-handles emanating from it. Thus there have to be at least two 0 -handles inside $D^{2}$, so that $K$ has at least three 2-cells. Since the handlebody closes up, $D^{2}-\mathcal{H}_{f}$ must have at least two components, resulting in at least two vertices in $K$. 


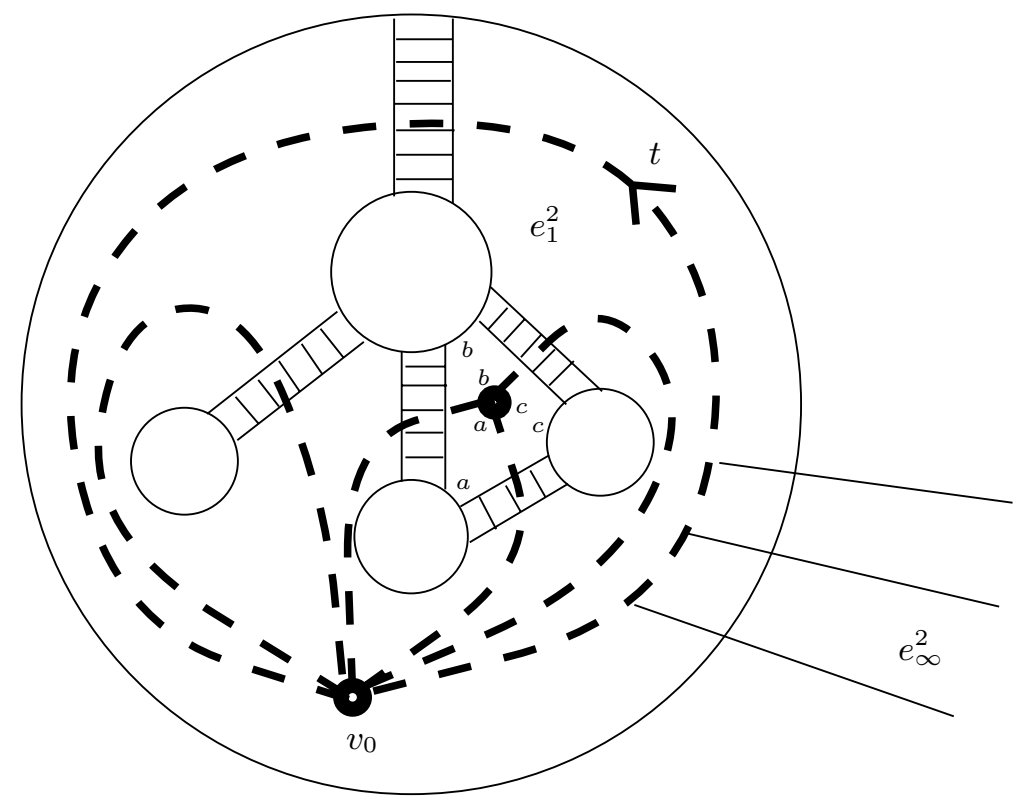

Figure 5: The cell complex $K$ (shown dashed)

\section{The key technical theorem}

In this section we prove the following result whose proof is modeled on that of [4; Theorem 4.1]. We show that the hypotheses of the cell subdivision lemma are self-contradictory.

\section{Key Technical Theorem}

Assume the working hypotheses. Then $g t$ is never in the kernel of the natural map $\Gamma *\langle t\rangle \rightarrow \widehat{\Gamma}$ for any $g \in \Gamma$.

Remark Assuming this theorem, note that by Lemma 1 in the Introduction, $\Gamma \rightarrow \widehat{\Gamma}$ is not surjective. Therefore by taking $H$ and $H^{\prime}$ to be trivial, we can now deduce a special case of our main theorem:

If the $t$-shape of $w=w_{0}$ is not $t$ (ie, $w$ is not conjugate to gt for any $g \in \Gamma$ ) but is of the form $t^{-1} t t^{-1} \ldots t t^{-1} t t$ then $q: \Gamma \rightarrow \widehat{\Gamma}$ is not surjective.

In the next section we introduce an algebraic trick which will enable us to deduce the general case, where $\operatorname{ex}(w)=1$ and $w$ is not conjugate to $g t$ for any $g \in G$, from this special case. 
Proof The proof relies heavily on the proof and terminology of [4; Theorem 4.1, pages 62-64]. Assume that $g t$ is in the kernel of $\Gamma *\langle t\rangle \rightarrow \widehat{\Gamma}$ where $g \in \Gamma$. By the cell subdivision lemma there is a cell subdivision of $S^{2}$ with all 2-cells of the four types $I, I^{\prime}, I I, I I^{\prime}$ illustrated in Figure 6 with the exception of the special 2-cell $e_{\infty}^{2}$.
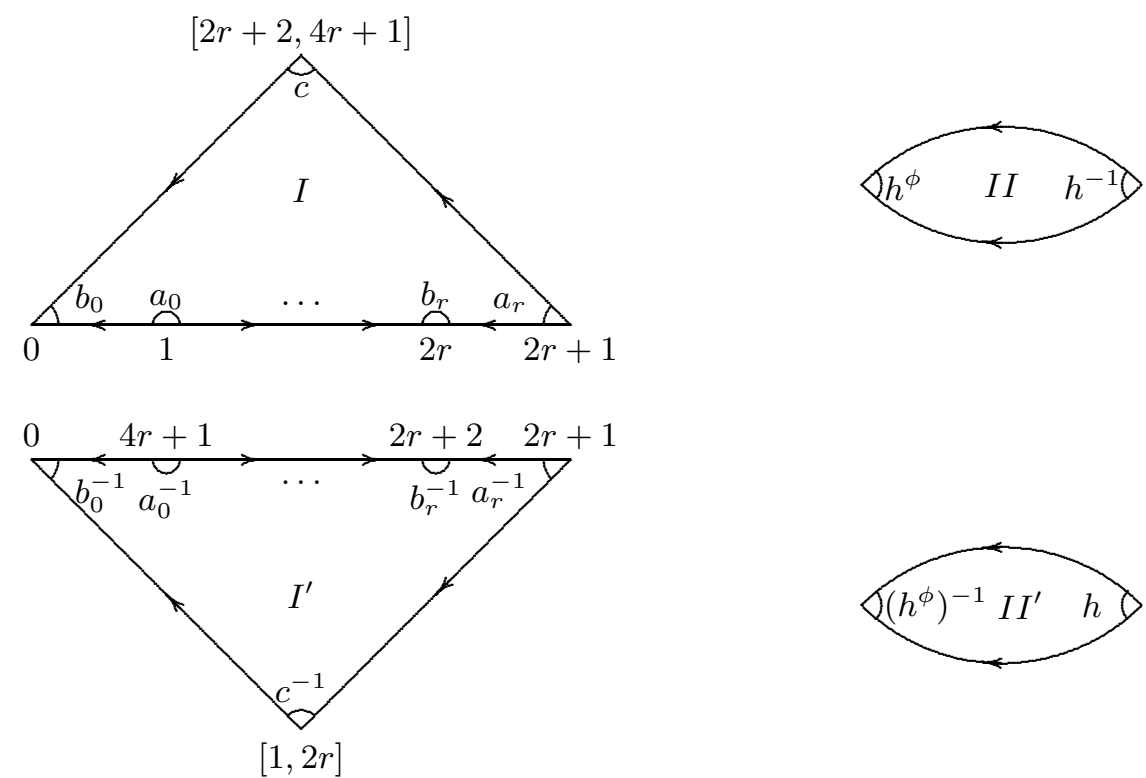

Figure 6: The 2-cells $I, I^{\prime}, I I$ and $I I^{\prime}$

As in [4] we give the two-sphere an orientation ("anticlockwise") and give each 2 -cell of $K$ the induced orientation. A traffic flow is now defined, with a car running around the boundary of each 2 -cell in the direction of the induced orientation as follows:

At time 0 let a car on the boundary of a country of type $I$ or $I^{\prime}$ start at the corner labelled $b_{0}$ or $b_{0}^{-1}$ and proceed in an anticlockwise manner with respect to the orientation of the edge along which it is travelling, moving from corner to corner in unit time except at the corner labelled $c$ or $c^{-1}$ where it stops for $2 r-1$ units. The times when the car is at each corner are illustrated in figure 6. For countries of type $I I$ or $I I^{\prime}$ the car starts at the corner labelled $h^{\phi}$ or $\left(h^{\phi}\right)^{-1}$ and proceeds in an anticlockwise manner moving from corner to corner in unit time.

For $e_{\infty}^{2}$ we need to consider also the 2-cell $e_{1}^{2}$ whose boundary properly contains the boundary of $e_{\infty}^{2}$, see figure 5 . Let $A$ be the car on the boundary of $e_{\infty}^{2}$ and $B$ the car on the boundary of $e_{1}^{2}$. Choose a point $\omega$ on $\partial e_{\infty}^{2}$ different from $v_{0}$. 
We shall engineer crashes between $A$ and $B$ to occur precisely at $\omega$. Suppose that $B$ is approaching $\omega$ then let $A$ approach $\omega$ from the opposite direction to crash at $\omega$. After the crash, let $A$ dawdle near $\omega$ until $B$ moves off $\partial e_{\infty}^{2}$ (which it must, because $\partial e_{1}^{2}$ properly contains $\partial e_{\infty}^{2}$ ); then let $A$ speed round to just before $\omega$ where it again dawdles until $B$ again approaches $\omega$ at which point the cycle repeats.

Recall from [4] that a complete car crash is said to occur when two cars meet in the interior of an edge (necessarily going in opposite directions) or when a $N$ cars from $N$ neighbouring countries all meet at a vertex of valency $N$.

Notice that, on $\partial e_{\infty}^{2}$, the given flow has the property that complete crashes occur at $\omega$ and nowhere else; in particular, no complete crash occurs at $v_{0}$. However there must be another complete crash occurring at some other vertex of $K$. (This is for exactly the same reasons as in the proof of [4; Theorem 4.1]. Properies 1 to 4 on pages 63-64 hold here also and the flow satisfies the conditions of the Crash Theorem with Stops [4, Theorem 2.3, page 56]. So there must be another complete crash and, as in [4], this must occur at a vertex.)

This leads to the identical contradiction as on [4; page 64]: The flow has been chosen so that, at a vertex where all the cars come together at the same time, the labels around the corners are all $\left\{a, a^{-1}\right\}$ for some coefficient $a=a_{i}$ of $w_{0}$ together with elements of $H$ or $\left\{b, b^{-1}\right\}$ for some coefficient $b=b_{i}$ of $w_{0}$ together with elements of $H^{\prime}$. For definiteness assume that we are in the former situation. Then we can read an (unreduced) word of the form $a^{\epsilon} h_{1} h_{2} \ldots h_{i_{1}} a^{\epsilon} h_{1} h_{2} \ldots h_{i_{2}} a^{\epsilon} \ldots$ which is 1 in $\Gamma$. Now if this word contains a subword of the form $a^{\epsilon} a^{-\epsilon}$ then $K$ is type (1) reducible and if it contains a subword of the form $h_{1} h_{2} \ldots h_{i}$ which is 1 in $\Gamma$ then $K$ is type (2) reducible. Since $K$ is irreducible neither of these happen and the word either gives a nontrivial relation in $\langle a, H\rangle$ contradicting the assumption that $a$ is free relative to $H$ or reads $\left(a^{\epsilon}\right)^{N}=1$ for $N \geq 1$ which also contradicts the assumption that $a$ is free relative to $H$ (and in particular has infinite order).

\section{Proof of the main theorem}

In the light of the discussion in the Introduction and Lemma 1, we assume that $\operatorname{ex}(w)=1$ and, proving the contrapositive of the Main Theorem, we assume that $w$ is not conjugate to $g t$ for any $g \in G$. We must prove that

$$
g t \text { is not in the kernel of } \pi: G *\langle t\rangle \longrightarrow \widehat{G} \text { for any } g \in G \text {. }
$$

We now use Klyachko's algebraic trick described on pages $64-66$ of [4]. 
Consider the homomorphism ex: $G *\langle t\rangle \rightarrow \mathbb{Z}$. It is well known that $K$, the kernel of ex, is a free product of copies of $G$ generated by elements of the form $g^{t^{\mathcal{O}}}=t^{-\mathcal{O}} g t^{\mathcal{O}}, 1 \neq g \in G$.

Any element of $K$ has a canonical expression of the form $k=g_{1}^{t^{\mathcal{O}_{1}}} \cdots g_{r}^{t^{\mathcal{O}_{r}}}$, where $\mathcal{O}_{i} \neq \mathcal{O}_{i+1}$ for each $i$. We shall call the $g_{i}^{t^{\mathcal{O}_{i}}}$ the canonical elements of $k$. Let $\min (k)$ be the minimum value of $\mathcal{O}_{i}, i=1, \ldots, r$ and $\max (k)$ the maximum value. Fix a positive integer $m$. Consider the following subgroups of $K$ :

$$
\begin{aligned}
H & =\langle k \in K \mid \min (k) \geq 0, \max (k) \leq m-2\rangle \\
H^{\prime} & =\langle k \in K \mid \min (k) \geq 1, \max (k) \leq m-1\rangle \\
J & =\langle k \in K \mid \min (k) \geq 0, \max (k) \leq m-1\rangle
\end{aligned}
$$

and the following subsets:

$$
\begin{aligned}
& X=\{k \in K \mid \min (k)=0, \max (k) \leq m-1\} \\
& Y=\{k \in K \mid \min (k) \geq 0, \max (k)=m-1\} \\
& Z=\{k \in K \mid \min (k) \geq 1, \max (k)=m\} .
\end{aligned}
$$

Lemma 2 [4; Lemma 4.2, page 65] Let $w \in G *\langle t\rangle$ satisfy $\operatorname{ex}(w)=1$. Then, after conjugation, $w$ can be written as a product

$$
b_{0} a_{0}^{t} b_{1} a_{1}^{t} \cdots b_{r} a_{r}^{t} c t
$$

where $a_{i} \in Y, b_{i} \in X, i=0, \ldots, r$ and $c \in J$ for some $m>0$.

Furthermore, provided $w$ is not conjugate to $g t$ for some $g \in G$, then $r \geq 0$ in the expression.

Remark The final sentence in the statement of lemma 2 is not given in [4], but is immediate from the proof given there.

Lemma 3 [4; Lemma 4.3, page 66] Suppose that $G$ is torsion-free, then any element $a$ of $Y$ is free relative to $H$. Similary any element $b$ of $X$ is free relative to $H^{\prime}$.

We can now complete the proof of the main theorem, which follows closely the proof of [4; Theorem 4.4, pages 66-67]. By lemma 2 we can assume that $w=b_{0} a_{0}^{t} b_{1} a_{1}^{t} \cdots b_{r} a_{r}^{t} c t$, where $a_{i} \in Y, b_{i} \in X, i=0, \ldots, r$ and $c \in J$ and $r \geq 0$. We need to think of each $a_{i}, b_{i}, c$ as functions of $t$ and for clarity we shall introduce a new variable $s$. To be precise let

$$
w(s, t) \equiv b_{0}(t) a_{0}^{s}(t) \cdots b_{r}(t) a_{r}^{s}(t) c(t) s
$$


where $s$ and $t$ are independent variables.

Write $\Gamma$ for $G *\langle t\rangle$ and let $H, H^{\prime}$ be the subgroups defined above. There is an isomorphism $\phi: H \rightarrow H^{\prime}$ given by $h^{\phi}=h^{t}, h \in H$.

Lemma 3 gives the hypothesis of our key technical theorem in Section 4, which implies that

$$
g s \text { is never in the kernel of } \Gamma *\langle s\rangle \rightarrow \widehat{\Gamma} .
$$

The case $m=1$ is the special case (with $t$-shape $t^{-1} t t^{-1} \ldots t t^{-1} t t$ ) covered by the proof in the last section, so we may assume that $m>1$ and then $G \subset H \neq \emptyset$.

Each of the canonical elements of $a_{i}(t), b_{i}(t), c(t)$ is either in $G$ or lies in $H^{t}$; moreover in $\widehat{\Gamma}$ we have $h^{s}=h^{\phi}=h^{t}$ for each $h \in H$.

Since $H$ is generated by elements of the form $t^{-i} g t^{i}$ for $i \leq m-2$ and since $h^{s}=h^{t}$ for each $h \in H$ it follows by induction on $i$ that we can freely exchange $s$ and $t$ in products of elements of the form $t^{-i} g t^{i}$ for $i \leq m-1$. Thus we can exchange $s$ and $t$ in the coefficients of $w(s, t)$ and it follows that $w(s, s)=1$ in $\widehat{\Gamma}$.

Now consider the following commutative diagram

$$
\begin{array}{ccccc}
\Gamma=G *\langle t\rangle & \subset & \Gamma *\langle s\rangle & \longrightarrow & \widehat{\Gamma} \\
\cup & & \cup & & \uparrow \\
G & \longrightarrow & G *\langle s\rangle & \longrightarrow & \widehat{G}
\end{array}
$$

where $\widehat{G}=\frac{G *\langle s\rangle}{\langle\langle w\rangle}$.

By $(* *), g s \in \Gamma *\langle s\rangle$ does not map to $1 \in \widehat{\Gamma}$ for any $g \in G$. Therefore $g s \in G *\langle s\rangle$ never maps to $1 \in \widehat{G}$. This proves $(*)$ as required.

\section{The surjectivity problem and Whitehead torsion}

If one adds $n$ generators $x_{1}, x_{2}, \ldots x_{n}$ and $n$ relators $w_{1}, w_{2}, \ldots w_{n}$ to a group $G$ to form the group $\widehat{G}$ then one can ask whether the natural homomorphism $G \longrightarrow \widehat{G}$ is injective. If it is injective then one can ask whether it is surjective. Our main theorem answers this question completely for torsion-free groups when $n=1$.

The question of surjectivity, assuming injectivity, was raised by Cohen [2] in his study of Zeeman's conjecture. Assuming the natural homomorphism is injective then one can associate (after some normalization - see [9, pages 600-601]) 
to the set of words $w_{1}, \ldots w_{n}$ a Whitehead torsion element $\tau \in \mathrm{Wh}(G)$ (the Whitehead group of $G$ ). Cohen conjectured that if $\tau \neq 0$ then the injection cannot be onto. In closely related but independent work, in which he investigated inclusions of one 2-complex into another which are homotopy equivalences, Metzler [6] investigated the group theoretic combinatorics and the set of Whitehead torsion elements which are associated to such homotopy equivelences. He named the set of Whitehead torsion elements which can be realized by a relative 2 -complex as $\mathrm{Wh}^{*}(G)$

Our main theorem appears to give evidence that $\mathrm{Wh}^{*}(G)=0$. In fact, when $n=1$ not only do we show that a necessary condition for surjectivity is that the torsion of the $1 \times 1$ matrix is 0 , but we show that, up to homotopy of the attaching map, the added one- and two- cells can be collapsed away. However, one must be very cautious concerning what this means for $n>1$ in that

- not all Whitehead torsion elements can be realized by $1 \times 1$ matrices, hence our result for $n=1$ in no way answers the question of whether $\mathrm{Wh}^{*}(G)=0$,

- it is possible (an open conjecture) that $\mathrm{Wh}(G)=0$ for all torsion-free groups.

The only significant results on the surjectivity problem which we know of for $n>1$ are those of Rothaus [9]. He develops an obstruction to the surjectivity of the map $G \longrightarrow \widehat{G}$ in terms of representations of $G$ into compact connected Lie groups. His theory had the following application for dihedral groups, whose Whitehead groups are known to be non-trivial.

Theorem [9; Theorem 11] If $\mathrm{p} \geq 5$ is an odd prime and $G=D_{2 p}$ is the dihedral group of order $2 p$ and $n$ is any positive integer then there exist non-trivial Whitehead torsion elements such that every injective homomor-

phism $G \longrightarrow \widehat{G}=\frac{G *\left\langle t_{1}, \ldots t_{n}\right\rangle}{w_{1} \ldots w_{n}}$ realizing this Whitehead torsion element is nonsurjective.

Beyond Rothaus' work, the surjectivity problem for $n>1$ is an open and fascinating question.

\section{An extension and a question}

The main theorem (in the equivalent form given by lemma 1) can be extended: 


\section{Extension of Main Theorem}

Let $G$ be a torsion free group and let $w \in G *\langle t\rangle$ be word which is not of the form $g t$ and whose $t$-shape is amenable (see $[4,5]$ ) and consider the natural map $\pi: G \rightarrow \widehat{G}=\frac{G *\langle t\rangle}{\langle\langle w\rangle\rangle}$. Let $x \in G *\langle t\rangle$ be any word with $t$-shape $t^{n}$ for some $n>0$.

Then $x$ is not in the kernel of $\pi$.

The proof is very similar to the proof of the main theorem. The cell $e_{\infty}^{2}$ has $n$ edges all oriented the same way ("uphill"). Notice that any other cell with an edge in common with $e_{\infty}^{2}$ has its car traverse that edge in the "downhill" direction, since adjacent cells induce opposite orientations on a common edge. Choose any point $\omega \in \partial e_{\infty}^{2}$ not at a vertex. The flow constructed as in [4; page $68]$ for cells other than $e_{\infty}^{2}$ has the property that there are times when all cars are going uphill and hence are not on $\partial e_{\infty}^{2}$. This leaves time for car $A$ (on $\partial e_{\infty}^{2}$ ) to rush round from just after $\omega$ to just before and hence there are no complete crashes on $\partial e_{\infty}^{2}$ except at $\omega$. This leads to the identical contradiction as in the proof of the main theorem.

The extension implies that all words of $t$-shape $t^{n}$ for some $n$ have infinite order in $\widehat{G}$. This leads to the natural question:

Question Suppose that $G$ is torsion-free and that $w$ is an amenable word. Is $\widehat{G}$ torsion-free?

If the answer is yes, then we can deduce that $G \rightarrow \widehat{G}$ is never surjective when $\widehat{G}$ is obtained from $G$ by adding $n$ generators and $n$ relators one pair at a time.

\section{References}

[1] M M Cohen, A Course in Simple-Homotopy Theory, GTM 10, SpringerVerlag, New York (1973)

[2] M M Cohen, Whitehead torsion, group extensions, and Zeeman's conjecture in high dimensions, Topology, 16 (1977) 79-88

[3] Marshall M Cohen, Martin Lustig, The conjugacy problem for Dehn twist automorphisms of free groups, Comment. Math. Helv. 74 (1999) 179-200

[4] Roger Fenn, Colin Rourke, Klyachko's methods and the solution of equations over torsion-free groups, L'Enseignment Mathématique, 42 (1996) 49-74

[5] Roger Fenn, Colin Rourke, Characterisation of a class of equations with solutions over torsion-free groups, from: "The Epstein Birthday Schrift", (I Rivin, 
C Rourke and C Series, editors), Geometry and Topology Monographs, Volume 1 (1998) 163-17

[6] W Metzler, Über den Homotopietyp zweidimensionaler $C W$-Komplexe und Elementartransformationen bei Darstellungen von Gruppen durch Erzeugende und definierende Relationen, J. Reine Angew. Math. 285 (1976) 7-23

[7] M Kervaire, On higher dimensional knots, Differential and combinatorial topology - a symposium in honour of Marston Morse, Princeton Math. Series, 27 (1965)

[8] A Klyachko, Funny property of sphere and equations over groups, Comm. in Alg. 21 (1993) 2555-2575

[9] O S Rothaus, On the non-trivialty of some group extensions given by generators and relations, Ann. of Math. 106 (1977) 599-612

[10] C P Rourke, B J Sanderson, Introduction to piecewise-linear topology, Springer study edition, Springer-Verlag, Berlin (1982) 